\title{
Is there equal pay in healthcare? Not if you are a doctor
}

The figures in this Feature by John Appleby (BMJ 2012;345:e6191, doi:10.1136/bmj.e6191) got muddled so that figure 2 appeared with the legend for figure 1 and vice versa. Furthermore, in the online version, to understand figure 2 the keys for "Nursing auxiliaries and assistants" and "Medical practitioners" should be transposed; these were mistakenly swapped during the editing process.

Cite this as: BMJ 2012;345:e6441

๑ BMJ Publishing Group Ltd 2012 